\title{
Ratio of T1-Weighted to T2-Weighted Signal Intensity as a Measure of Tissue Integrity: Comparison with Magnetization Transfer Ratio in Patients with Multiple Sclerosis
}

\author{
(DD. Pareto, (D) A. Garcia-Vidal, (D) M. Alberich, (DC. Auger, (D) X. Montalban, (D) M. Tintoré, (D). Sastre-Garriga, and (D) À. Rovira
}

\section{ABSTRACT}

SUMMARY: The study aim was to compare the ratio of TIWI to T2WI signal intensity (T1/T2) with magnetization transfer ratio, a marker of myelin integrity, in patients with multiple sclerosis. A moderate correlation $(r=0.50, P=.034)$ was found between the magnetization transfer ratio and T1/T2 in normal-appearing gray matter, and a strong correlation for normal-appearing white matter $(r=0.63, P=.005)$ and lesions $(r=0.70, P=.001)$. Results suggest that besides myelin integrity, other factors may be playing a role in $\mathrm{T} 1 / \mathrm{T} 2$ measures.

ABBREVIATIONS: EDSS = Expanded Disability Status Scale; MTR = magnetization transfer ratio; NAGM = normal-appearing gray matter; NAWM = normal-appearing white matter; CSF = cerebrospinal fluid

$\mathbf{T}$ he ratio of T1WI to T2WI signal intensity (T1/T2) on MR imaging has been recently proposed as a measure of myelin integrity in the brain. ${ }^{1,2} \mathrm{~T} 1 / \mathrm{T} 2$ has been investigated in multiple sclerosis, ${ }^{3-5}$ though no conclusive validation of the T1/T2 pathologic substrate has been established. This method has the advantage of being easily obtained and available, a feature that enables analysis of retrospective cohorts with considerable clinical value. $\mathrm{T} 1 / \mathrm{T} 2$ has been compared with the magnetization transfer ratio (MTR) ${ }^{4}$ considered a measure of tissue integrity related to myelin content, ${ }^{6}$ but only in specific portions of myelinated and demyelinated cortex in postmortem tissue samples of patients with MS. The aim of this study was to compare in vivo and regional T1/ T2 with MTR in a cohort of patients with MS. The comparison involved 2 regions, normal-appearing gray (NAGM) and white matter (NAWM) and the lesion mask derived from T2WI. An

Received November 5, 2019; accepted after revision January 5, 2020.

From the Neuroradiology Section (D.P., A.G.-V., M.A., C.A., A.R.), Department of Radiology and Department of Neuroimmunology-Cemcat (X.M., M.T., J.S.-G.), Vall d'Hebron University Hospital and Research Institute, Autonomous University, Barcelona, Spain; and Division of Neurology (X.M.), St. Michael's Hospital, University of Toronto, Toronto, Ontario, Canada.

This study was partly funded by the Spanish Multiple Sclerosis Network (Red Española de Esclerosis Múltiple) (RD07/0060, RD12/0032) and the project PI18/ 00823, which are sponsored by the Fondo de Investigación Sanitaria (Instituto de Salud Carlos III), by Financial Support for Research Groups in Catalonia (2009 SGR 0793), and by the Agency for Administration of University and Research Grants of the Generalitat de Catalunya in Spain.

Please address correspondence to Deborah Pareto, MD, Radiology Department, Vall Hebron University Hospital, Psg. Vall Hebron 119-129, Barcelona 08036, Spain e-mail: deborah.pareto.idi@gencat.cat

- Indicates open access to non-subscribers at www.ajnr.org

http://dx.doi.org/10.3174/ajnr.A6481 additional lesion-by-lesion comparison between the 2 measures was performed for each patient separately.

\section{MATERIALS AND METHODS \\ Patients}

Twenty-two patients with relapsing-remitting MS according to the 2017 McDonald criteria were included in this exploratory study. Clinical variables, the Expanded Disability Status Scale (EDSS) score, and disease duration were obtained for each patient. The study was approved by the Vall Hebron University Hospital local ethics committee, and patients signed informed consent.

\section{MR Imaging Acquisition}

Images were acquired on a $3 \mathrm{~T}$ scanner (Tim Trio; Siemens, Erlangen, Germany) with a 12-channel phased array head coil and a whole-body transmit coil. The protocol included the following: 1) 3D T1WI MPRAGE (TR $=2300 \mathrm{~ms}$; TE $=2.98 \mathrm{~ms}$; FOV $=256 \times 256 \mathrm{~mm} ; 192$ sections; voxel size $=1 \times 1 \times 1$ $\mathrm{mm})$; 2) $2 \mathrm{D}$ dual-echo T2WI $(\mathrm{TR}=2500 \mathrm{~ms}$; TE $=16 / 91 \mathrm{~ms}$; $\mathrm{FOV}=256 \times 256 ; 46$ sections; voxel size $=0.78 \times 0.78 \times$ $3.0 \mathrm{~mm}$ ); and 3) 2D gradient-echo magnetization transfer performed without $(\mathrm{TR}=1500 \mathrm{~ms} ; \mathrm{TE}=10 \mathrm{~ms} ; \mathrm{FOV}=250 \times 250$ $\mathrm{mm}$; 50 sections; voxel size $=1 \times 1 \times 3 \mathrm{~mm}$; flip angle $\left.=20^{\circ}\right)$ and with magnetization transfer saturation (equivalent flip angle $=500^{\circ}$; off-water resonance $=1.2 \mathrm{kHz}$; duration $=10 \mathrm{~ms}$ ).

\section{Image Analysis}

Lesion masks were outlined semiautomatically with Jim software (http://www.xinapse.com/home.php) on the proton-density 
T2WI, by a technician with $>10$ years' experience (M.A.), supervised by an experienced neuroradiologist (A.R.). The T1/T2 was generated after coregistering and reslicing the T2WI to the T1WI and bias-correcting the T1WI and T2WI with Statistical Parametric Mapping (SPM12; http://www.fil.ion.ucl.ac.uk/spm/software/spm12). The NAGM, NAWM, and cerebrospinal fluid (CSF) compartment masks were obtained after segmenting the T1WI with SPM12 and thresholding $(P>.75)$ and multiplying them by the inverse of the lesion mask to remove any contributions due to lesion misclassification. The MTR image was calculated as follows: $\left(\mathrm{MT}_{\text {off }}-\mathrm{MT}_{\text {on }}\right) / \mathrm{MT}_{\text {off }}$ and it was coregistered to T1/T2 with SPM12. Finally, for each patient, mean values in NAGM, NAWM, the lesion mask, and each lesion separately (after splitting the lesion mask) were computed from the MTR and T1/T2 images.

\section{Statistical Analysis}

To assess the relationship between MTR and T1/T2, we ran partial correlations for NAGM, NAWM, CSF, and the lesion mask, with age and sex as covariates. In addition, a correlation for each patient was obtained after comparing their individual lesions in MTR and T1/T2. Partial correlations were also run to assess relationships between either MTR or T1/T2 and EDSS and disease duration. Significance for the comparisons was set at $P<.05$, and analyses were performed with the SPSS (IBM, Armonk, New York).

\section{RESULTS}

A total of 22 patients were analyzed (68\% women; mean age, $41.13 \pm 6.04$ years; mean disease duration, 5.66 years; range, 0.08-20 years; mean EDSS score, 2.47; range, 0-6; and mean

Mean values of MTR and $\mathrm{T} 1 / \mathrm{T} 2$ in the different compartments and partial correlations ${ }^{\mathrm{a}}$

\begin{tabular}{lccc}
\hline & Mean MTR (SD) & Mean T1/T2 (SD) & $\boldsymbol{r}(\boldsymbol{P}$ Value $)$ \\
\hline NAWM & $0.510(0.032)$ & $1.022(0.102)$ & $0.631(.005)$ \\
Lesions & $0.477(0.054)$ & $0.604(0.074)$ & $0.699(.001)$ \\
NAGM & $0.435(0.030)$ & $0.537(0.043)$ & $0.502(.034)$ \\
CSF & $0.267(0.028)$ & $0.110(0.043)$ & $0.631(.040)$ \\
\hline
\end{tabular}

${ }^{a}$ Age and sex are covariates. lesion volume, $5.02 \mathrm{~mL}$; range, $0.13-25.45 \mathrm{~mL})$. For both methods, the differences within compartments were significant $(P<$ .05 ) for all possible pairs (data not shown). Mean values and partial correlations between MTR and T1/T2 measures are reported in the Table. Lesion-by-lesion correlations were significant for cases with lesion volumes of $>1 \mathrm{~mL}$ (17 cases of 22), with correlations ranging from 0.42 to 0.99 . An individual example can be seen in the Figure. Both MTR $(r=-0.64, P=.004)$ and T1/T2 $(r$ $=-0.66, P=.003)$ mean values in the lesion mask correlated with the EDSS, whereas only the T1/T2 lesion mask values correlated with disease duration $(r=-0.52, P=.021)$.

\section{DISCUSSION}

This exploratory study compares the distribution in T1/T2 and MTR in a relatively small group of patients with relapsing-remitting MS. Healthy controls were not included in this study because the main goal was to investigate the correspondence between MTR and T1/T2 in damaged tissue. Comparisons were performed across subjects for NAGM, NAWM, and lesion masks and also across individual lesions in each subject. Several factors may have affected the lesion-by-lesion correlations, such as small coregistration errors between MTR and T1/T2, which may have displaced the lesion masks, as well as patient movement during acquisition.

The correlations ranged from moderate to strong, suggesting that most probably, the 2 methods are not sensitive to the same pathologic substrate. In addition, T1/T2 was associated with the EDSS and disease duration, while MTR was only associated with EDSS. The concept that T1/T2 is a proxy for myelin in gray matter has been derived from studies performed in healthy individuals, ${ }^{1,2}$ in whom neither myelin nor axonal loss nor inflammation is present. In MS, T1/T2 in gray matter has been related to dendrite density rather than myelin ${ }^{5}$ in a study performed ex vivo in tissue blocks from brain donors; and, again in an ex vivo study, T1/T2 has shown a moderate correlation with MTR in gray matter. ${ }^{4}$ However, it is expected that T1/T2 will also be affected by edema and iron content, because T1WI and T2WI separately are affected. ${ }^{3}$ MTR has been mainly related to both myelin and axonal damage and, to a lesser degree, to inflammation and gliosis in
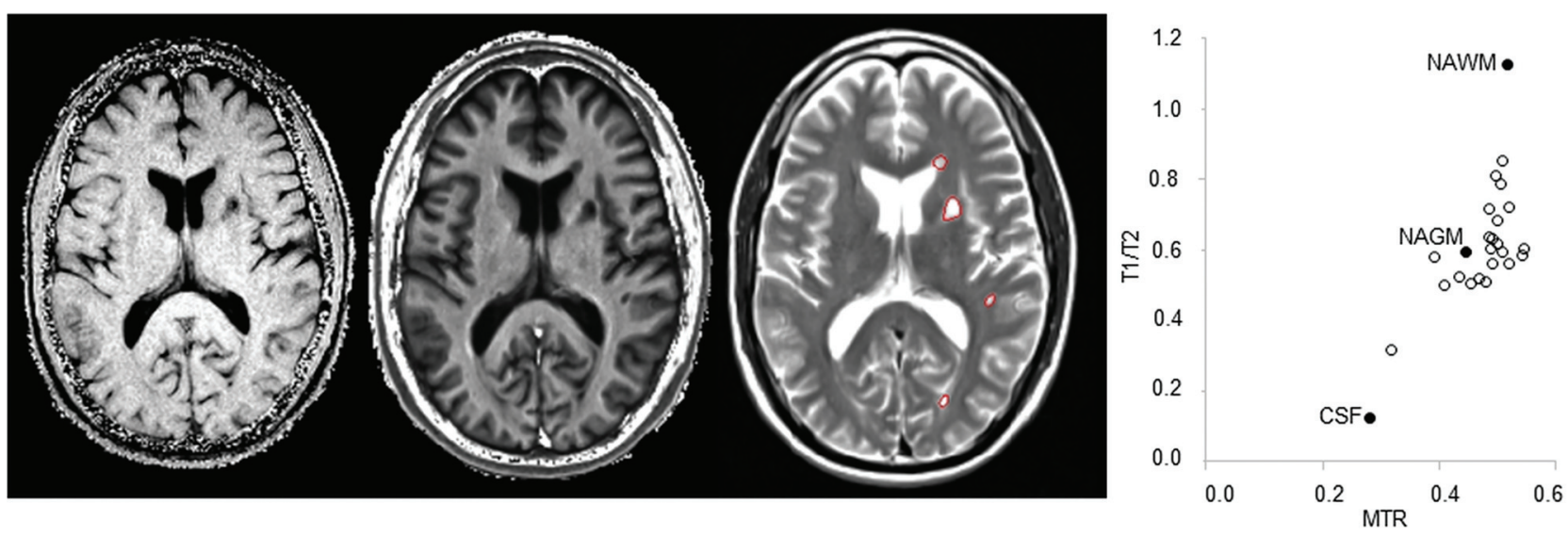

FIGURE. Representative MTR, T1/T2, and T2 with lesions outlined (left) and the corresponding scatterplot ( $r=0.630 ; P=.002 ; 22$ lesions; total volume $=3.39 \mathrm{~mL}$ ). Empty symbols refer to the individual lesions; filled symbols refer to the mean value in CSF, NAGM, and NAWM. 
MS. ${ }^{6}$ In this context, it is likely that compared with MTR, T1/T2 is more dependent on additional pathologic substrates and not just on myelin integrity, though new studies in vivo are needed to confirm the source of the signal in the T1/T2 approach. The main advantage of $\mathrm{T} 1 / \mathrm{T} 2$ is that it provides a wider range of values than those given by MTR, as was seen in postmortem studies. ${ }^{4}$ Thus, an intermediate degree of tissue destruction would be more likely to be captured with T1/T2 than MTR. Nevertheless, these results could not be extrapolated to any T1WI, T2WI, or MTR sequence because T1/T2 and MTR contrast and, consequently, the correlation between them, depends on the acquisition parameters. ${ }^{7}$ Further longitudinal studies with larger sample sizes as well as further in-depth assessment of lesional tissue (including black holes and new lesions) are warranted to assess the validity of T1/T2 as a biomarker of tissue damage and lesion recovery in MS.

\section{ACKNOWLEDGMENT}

We thank C. Cavallo for English writing support.

Disclosures: Deborah Pareto—_RELATED: Other: Novartis, Sanofi Genzyme, Comments: speaking honoraria, teaching activity, money paid to the individual. Xavier MontalbanUNRELATED: Consultancy: Actelion, Bayer AG, Biogen, Celgene, Genzyme, Merck, Novartis, Roche, Sanofi Genzyme, Teva Pharmaceutical Industries, EXCEMED, Multiple Sclerosis International Federation, and National Multiple Sclerosis Society. Jaume SastreGarriga - UNRELATED: Payment for Lectures Including Service on Speakers Bureaus: Genzyme, Biogen, Bayer AG, Merck, Almirall, Bial Pharmaceuticals, Novartis, Roche, Teva Pharmaceutical Industries, Celgene.* Alex Rovira - UNRELATED: Board Membership: Bayer AG, Biogen, Novartis, icometrix, Roche; Payment for Lectures Including Service on Speakers Bureaus: Bayer AG, Sanofi Genzyme, Bracco, Merck Serono, Teva Pharmaceutical Industries, Novartis, Roche, Biogen; Payment for Development of
Educational Presentations: Sanofi Genzyme, Merck Serono, Novartis, Roche, Biogen. Mar Tintoré-UNRELATED: Board Membership: Almirall, Bayer AG Schering Pharma, Biogen, Genzyme, Merck Serono, Novartis, Roche, Sanofi-Aventis, Teva Pharmaceutical Industries; Grants/Grants Pending: Biogen, Genzyme, Novartis*; Payment for Lectures Including Service on Speakers Bureaus: Almirall, Bayer AG, Biogen, Genzyme, Merck Serono, Novartis, Roche, Sanofi-Aventis, Viela Bio, Teva Pharmaceutical Industries; Payment for Development of Educational Presentations: Biogen. *Money paid to the institution.

\section{REFERENCES}

1. Glasser MF, Van Essen DC. Mapping human cortical areas in vivo based on myelin content as revealed by T1- and T2-weighted MRI. $J$ Neurosci 2011;31:11597-616 CrossRef Medline

2. Ganzetti M, Wenderoth N, Mantini D. Whole brain myelin mapping using T1- and T2-weighted MR imaging data. Front Hum Neurosci 2014;8:671 CrossRef Medline

3. Beer A, Biberacher V, Schmidt $P$, et al. Tissue damage within normal appearing white matter in early multiple sclerosis: assessment by the ratio of T1- and T2-weighted MR image intensity. J Neurol 2016;263:1495-502 CrossRef Medline

4. Nakamura K, Chen JT, Ontaneda D, et al. T1-/T2-weighted ratio differs in demyelinated cortex in multiple sclerosis. Ann Neurol 2017; 82:635-39 CrossRef Medline

5. Righart R, Biberacher V, Jonkman LE, et al. Cortical pathology in multiple sclerosis detected by the T1/T2-weighted ratio from routine magnetic resonance imaging. Ann Neurol 2017;82:519-29 CrossRef Medline

6. Schmierer K, Scaravilli F, Altmann DR, et al. Magnetization transfer ratio and myelin in postmortem multiple sclerosis brain. Ann Neurol 2004;56:407-15 CrossRef Medline

7. Henkelman RM, Stanisz GJ, Graham SJ. Magnetization transfer in MRI: a review. NMR Biomed 2001;14:57-64 CrossRef Medline 